\title{
Recent advances in understanding carotenoid-derived signaling molecules in regulating plant growth and development
}

\author{
Li Tian* \\ Department of Plant Sciences, University of California, Davis, Davis, CA, USA
}

Carotenoids $\left(\mathrm{C}_{40}\right)$ are synthesized in plastids and perform numerous important functions in these organelles. In addition, carotenoids can be processed into smaller signaling molecules that regulate various phases of the plant's life cycle. Besides the relatively well-studied phytohormones abscisic acid (ABA) and strigolactones (SLS), additional carotenoid-derived signaling molecules have been discovered and shown to regulate plant growth and development. As a few excellent reviews summarized recent research on ABA and SLs, this mini review will focus on progress made on identification and characterization of the emerging carotenoid-derived signals. Overall, a better understanding of carotenoid-derived signaling molecules has immediate applications in improving plant biomass production which in turn will have far reaching impacts on providing food, feed, and fuel for the growing world population.

The University of Tokyo, Japan Kimberley Cathryn Snowden, The New Zealand Institute for Plant and Food Research Limited,

New Zealand

*Correspondence:

Li Tian,

Department of Plant Sciences, University of California, Davis, Mail Stop 3, 116 Asmundson Hall, Davis,

CA 95616, USA

Itian@ucdavis.edu

Specialty section:

This article was submitted to

Plant Biotechnology,

a section of the journal

Frontiers in Plant Science

Received: 13 July 2015 Accepted: 11 September 2015 Published: 24 September 2015

Citation:

Tian L (2015) Recent advances in understanding carotenoid-derived signaling molecules in regulating plant growth and development.

Front. Plant Sci. 6:790 doi: 10.3389/fpls.2015.00790

Plants harness solar energy through photosynthesis to support their growth, development, reproduction, and amassment of carbon and nutrients in storage tissues. Carotenoid pigments play indispensable roles in light harvesting complexes and reaction centers of the photosystems. In addition to their direct involvement in photosynthesis, carotenoids and their cleavage products (i.e., apocarotenoids) also participate in controlling the plant's life cycle via coordinating activities among different organelles, cells, tissues, and organs. Research progress on two carotenoidderived phytohormones, abscisic acid (ABA) and strigolactones (SLs), has been reviewed recently (Cutler et al., 2010; Chater et al., 2014; Seto and Yamaguchi, 2014; Waldie et al., 2014; Mittler and Blumwald, 2015). Here, we focus on several additional carotenoid derivatives that have been investigated for their functions in regulating chloroplast biogenesis, shoot and/or root development, as well as stress response.

\section{Carotenoid-derived Signals in Shoot and Root Development}

About a decade ago, allelic bypass 1 (bps1) mutants that lack leaf vasculature and trichomes, produce short roots and exhibit arrested shoot apical meristem (SAM) activities were isolated from ethyl methanesulfonate (EMS) mutagenized Arabidopsis grown at $16^{\circ} \mathrm{C}$ (Van Norman et al., 2004). The shoot and root phenotypes of bps 1 were alleviated when the mutant plants were grown at higher temperatures (Van Norman et al., 2004). Through elegant genetic and biochemical analyses of the bps1 mutant and wild type plants, the Sieburth group showed that a mobile 
bypass (bps) signal is generated in roots of wild type Arabidopsis plants, which regulates root and shoot development through root-to-shoot signal transmission (Van Norman et al., 2004). Since application of carotenoid biosynthesis inhibitors or introduction of the $p d s 1$ mutation (containing an impaired carotenoid biosynthetic enzyme phytoene desaturase/PDS) could partially rescue the abnormal bps 1 growth phenotype, it was hypothesized that bps could be a signal originated from carotenoids (Van Norman et al., 2004; Van Norman and Sieburth, 2007). Further molecular, biochemical and genetic examinations indicated that the bps signal is distinct from ABA and SLs; its production entails synthesis of $\beta$-carotene branch carotenoids but does not require the activity of a single carotenoid cleavage enzyme (9-cis-epoxycarotenoid dioxygenase/NCED or carotenoid cleavage dioxygenase/CCD) (Van Norman and Sieburth, 2007; Figure 1). Besides BYPASS1 (BPS1), two additional BPS genes (BPS2 and BPS3) were identified in Arabidopsis and the bps triple mutant (bps1bps2bps3) has aberrant cell divisions during early embryogenesis that result in defective SAM, root apical meristem (RAM), and vascular meristem (VM) (Lee et al., 2012). Such embryonic defects are not evident in bps 1 and less pronounced in the bps 1 bps 2 double mutant, suggesting that the three Arabidopsis BPS genes possess overlapping yet distinct functions in generating the bps signal (Lee et al., 2012). Analyses of the auxin response markers as well as localization and trafficking of the PIN1 auxin efflux transporter in bps mutants revealed that the auxin signaling pathway is not a primary target of the bps signal for regulation of plant development (Lee and Sieburth, 2012; Lee et al., 2012). Though the chemical structure of bps is currently unknown, progress has been made recently toward elucidation of the bps signal using a bioassay-based purification and identification scheme (Adhikari et al., 2013).

Another shoot development mutant, carotenoid chloroplast regulatory1 (ccr1), was identified by the Pogson group in a screen for Arabidopsis mutants defective in carotenoid and chloroplast regulation (Cazzonelli et al., 2009). Unlike bps1 that contains a lesion in a gene of unknown function, $c c r 1$ encodes a malfunctional histone methyltransferase Set Domain Group8 (SDG8) (Cazzonelli et al., 2009; Figure 1). As a result of the $\operatorname{ccr} 1$ mutation, histone methylation of chromatin around carotenoid isomerase (CRTISO, an enzyme that converts tetracis-lycopene to all-trans-lycopene) and CRTISO expression were reduced, which led to significantly decreased accumulation of lutein and increased levels of $\beta$-carotene branch xanthophylls (Park et al., 2002). The ccrl mutant exhibited more abundant rosette and cauline branching than wild type plants, yet, unlike the Arabidopsis shoot branching mutants deficient in SLs, the ccr1 mutant phenotype could not be suppressed by a root generated, transmissible signal when grafted to the wild type Arabidopsis root stock (Cazzonelli et al., 2009). Though changes in carotenoid content were observed in $c c r 1$, it remains to be elucidated whether increased shoot branching in this mutant is indeed associated with a carotenoid-derived signal.

In addition to mutant analysis (e.g., bps1, ccr1) that allows efficient identification of altered plant growth and the corresponding causal mutations, chemical treatment of

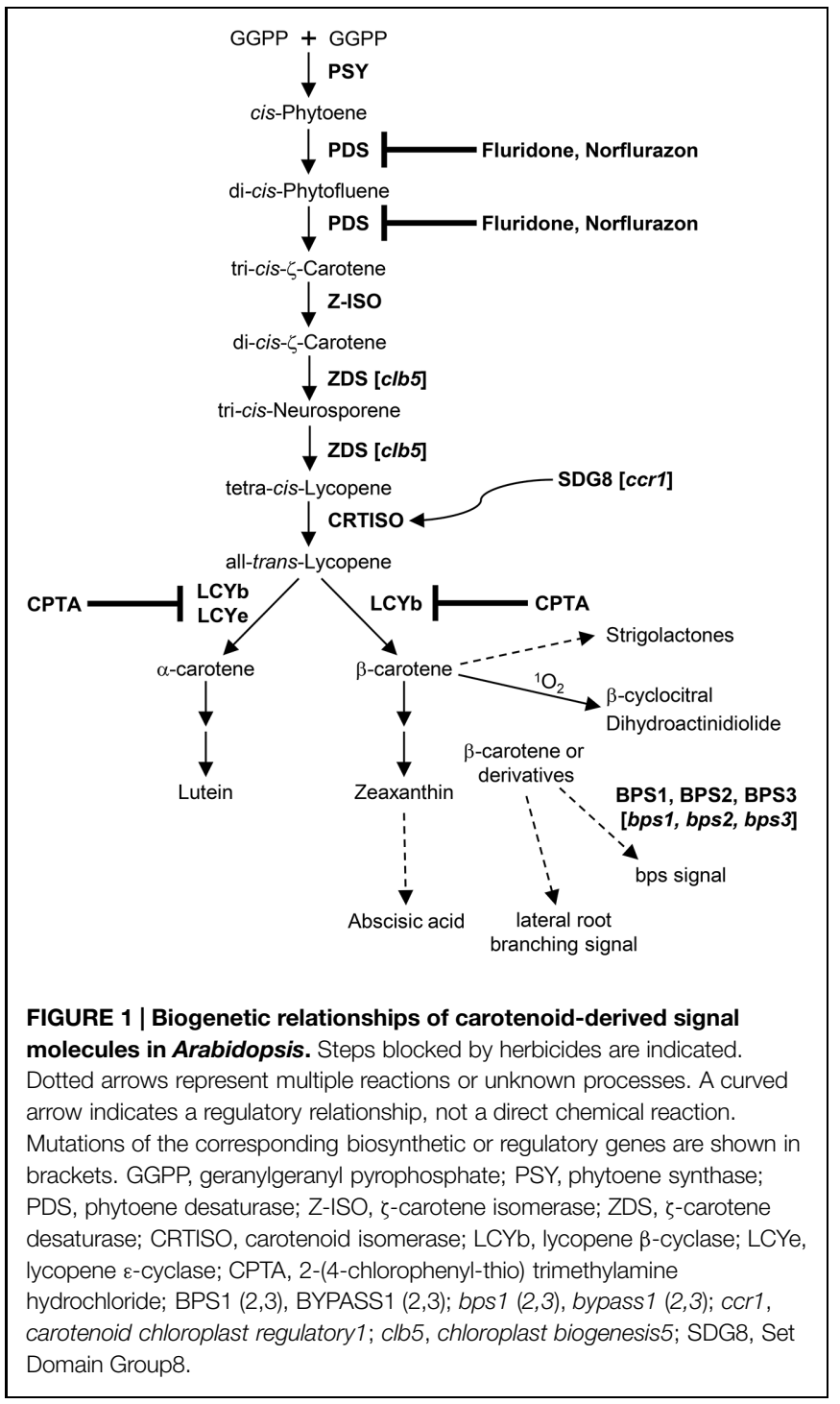

wild type Arabidopsis seedlings has also been valuable in uncovering a carotenoid-derived signal that controls lateral root (LR) branching. Van Norman et al. (2014) measured the root's capacity for LR formation (i.e., LR capacity) based on the number of prebranch sites and observed a decrease in LR capacity in Arabidopsis seedlings treated with carotenoid biosynthesis inhibitors, norflurazon (NF) and 2-(4-chlorophenyl-thio) trimethylamine hydrochloride (CPTA). Further support for association of reduced LR capacity with carotenoid deficiency was provided by application of NF and CPTA to Arabidopsis expressing a transcriptional reporter of auxin response, $p D R 5: L U C$; it was shown that inhibition of carotenoid production disturbed the rhythmic oscillation of the LR clock necessary for establishment of prebranch sites (Van Norman et al., 2014). Since ABA or SL deficiency does not impose a similar impact on LR capacity as carotenoid biosynthesis inhibitor treatment, a novel carotenoid-derived signal in LR determination and development has been proposed. 
Generation of this signal appears to require a CCD activity as treatment of Arabidopsis seedlings with a CCD inhibitor D15 led to significantly decreased LR capacity (Van Norman et al., 2014). Since D15 treatment, which blocks CCD activities and prevents carotenoid turnover, caused substantially more increases in root carotenoid content than in shoots, the authors hypothesized that D15 could inhibit a root-specific CCD activity. However, it is also possible that D15 could be inhibiting the same CCD activity in both roots and shoots. This is because if D15 is a competitive inhibitor for carotenoids binding to CCD enzymes, it is conceivably more effective in roots that contain low levels of carotenoids than in shoots where abundant carotenoid accumulation is found. Understanding the mechanistic basis of D15 inhibition of CCD activities will help discern these possibilities. On another note, it will be interesting to evaluate the impact of overexpressing a root-specific CCD1 or CCD4 on LR capacity in the future.

\section{Carotenoid-derived Signals in Chloroplast Biogenesis, Leaf Development, and Stress Response}

While enzymatic cleavage by NCEDs or CCDs is necessary for production of several apocarotenoid signals (e.g., ABA and SLs), an exciting new line of research by the Havaux group indicated that chemical oxidation of $\beta$-carotene by singlet oxygen $\left({ }^{1} \mathrm{O}_{2}\right)$ can result in a series of short chain, cleavage derivatives of $\beta$-carotene, which are collectively designated carotenoid reactive electrophile species (RES) [e.g., $\beta$-cyclocitral $\left(\beta-\mathrm{CC}, \mathrm{C}_{7}\right)$ and dihydroactinidiolide ( $\left.\mathrm{dhA}, \mathrm{C}_{11}\right)$ ] (Ramel et al., 2012a,b, 2013a,b,c; Havaux, 2014; Shumbe et al., 2014). In Arabidopsis plants exposed to high light, oxidative cleavage of $\beta$-carotene by ${ }^{1} \mathrm{O}_{2}$ produces $\beta$-CC and $\mathrm{dhA}$, which elicit ${ }^{1} \mathrm{O}_{2}$ responsive gene expression in the nucleus via a retrograde signaling mechanism and subsequently lead to high light acclimation of the plants (Ramel et al., 2012b; Shumbe et al., 2014). Based on the highly specific impact of carotenoid RES on the ${ }^{1} \mathrm{O}_{2}$, but not the $\mathrm{H}_{2} \mathrm{O}_{2}$ marker gene expression, it was suggested that they facilitate plant acclimation to high light stress via the ${ }^{1} \mathrm{O}_{2}$ signaling pathway and is independent of $\mathrm{H}_{2} \mathrm{O}_{2}$. Carotenoid RES regulation also differs from the EXECUTER1 protein mediated programmed cell death response to ${ }^{1} \mathrm{O}_{2}$ as the executer 1 mutant plants could still acclimate to high light stress and respond to $\beta$-CC treatment in a similar way as wild type Arabidopsis (Ramel et al., 2012b). Interestingly, high light stress acclimation in the Arabidopsis chlorophyll $b$ deficient mutant $c h 1$ and the double mutant between $\operatorname{ch} 1$ and $d d e 2$ [a mutant deficient in jasmonate (JA)] is accompanied by a suppression of JA biosynthesis and decreased JA accumulation in leaves, suggesting an interaction between the carotenoid RES induced ${ }^{1} \mathrm{O}_{2}$ response and JA signaling pathways (Ramel et al., 2012b, 2013a,b). It further suggests that high light acclimated plants may be somewhat compromised for JA-mediated responses to pathogens and herbivores. Since ${ }^{1} \mathrm{O}_{2}$ could also be generated in plant defense against microbial pathogens (Triantaphylidès and Havaux, 2009), it will be informative to explore whether carotenoid RES signals also directly participate in biotic stress responses by plant hosts.

Besides the carotenoid RES control of nuclear gene expression, involvement of $\zeta$-carotene desaturase (ZDS) precursors in retrograde signaling was also proposed upon characterization of an Arabidopsis mutant of ZDS (Dong et al., 2007). After a close examination of null Arabidopsis zds/chloroplast biogenesis5 (clb5) mutant alleles, abnormal leaf development and cell differentiation as well as compromised auxin responses were also apparent in clb5, in addition to the albino phenotype common to mutants that lack a functional ZDS enzyme (Avendaño-Vázquez et al., 2014). Consistent with the report by Dong et al. (2007), the clb5 mutation modified expression of plastid- and nuclear-encoded genes involved in carotenoid biosynthesis and chloroplast development (Avendaño-Vázquez et al., 2014). Application of an inhibitor of the PDS activity fluridone or introduction of the $p d s 3$ mutation rescued the clb5 mutant gene expression and leaf development phenotypes, suggesting that products of the PDS reaction and precursors of the ZDS reaction, including dicis-phytofluene and $\zeta$-carotene isomers $(\zeta$-carotene isomerase $/ Z$ ISO catalyzes the isomerization of tri-cis- $\zeta$-carotene to di-cis- $\zeta-$ carotene), may be substrates for the underlying signals regulating chloroplast biogenesis and leaf development (Avendaño-Vázquez et al., 2014; Figure 1). Moreover, cleaved signals could be generated from di-cis-phytofluene and/or $\zeta$-carotene isomers by CCD4 as ccd4 mutation in the clb5 mutant background was able to rescue the clb5 mutant leaf phenotypes. Exogenous application of ABA or SLs did not affect the appearance of clb5 leaves, providing further support for novel carotenoid-derived signals controlling chloroplast and leaf development (AvendañoVázquez et al., 2014). It remains to be investigated whether these signals are generated locally in leaves or they could be mobile signals transported from roots.

\section{Perspectives}

Overall, substantial progress has been made on identification and characterization of carotenoid-derived signaling molecules in plants. With the exception of ABA, SLs, $\beta$-CC, and dhA, a primary unaddressed question regarding these carotenoidderived signals is their chemical identities. Aside from structure elucidation, there are several additional questions that remain to be answered: (1) How are the structurally uncharacterized apocarotenoid signals produced? Biochemical and genetic analyses suggested that carotenoid precursors could be subjected to catalysis by carotenoid cleavage enzymes. However, direct evidence for involvement of a single or multiple CCD enzymes is still lacking. (2) How are the carotenoid-derived signals transported between different cell types, tissues, and organs? (3) Thus far, work on carotenoids-derived signals, other than ABA and SLs, has been mainly conducted in Arabidopsis. Do these signals perform similar functions in other plants? (4) Do overlapping activities or antagonistic effects exist among different carotenoid-derived signaling molecules? (5) Are there additional carotenoid-derived signals remaining to be discovered? 


\section{References}

Adhikari, E., Lee, D.-K., Giavalisco, P., and Sieburth, L. E. (2013). Long-distance signaling in bypass 1 mutants: Bioassay development reveals the bps signal to be a metabolite. Mol. Plant 6, 164-173. doi: 10.1093/mp/sss129

Avendaño-Vázquez, A.-O., Cordoba, E., Llamas, E., San Román, C., Nisar, N., De la Torre, S., et al. (2014). An incharacterized apocarotenoid-derived signal generated in $\zeta$-carotene desaturase mutants regulates leaf development and the expression of chloroplast and nuclear genes in Arabidopsis. Plant Cell 26, 2524-2537. doi: 10.1105/tpc.114.123349

Cazzonelli, C., Cuttriss, A., Cossetto, S., Pye, W., Crisp, P., Whelan, J., et al. (2009). Regulation of carotenoid composition and shoot branching in Arabidopsis by a chromatin modifying histone methyltransferase, SDG8. Plant Cell 21, 39-53. doi: $10.1105 /$ tpc. 108.063131

Chater, C. C. C., Oliver, J., Casson, S., and Gray, J. E. (2014). Putting the brakes on: abscisic acid as a central environmental regulator of stomatal development. New Phytol. 202, 376-391. doi: 10.1111/nph.12713

Cutler, S. R., Rodriguez, P. L., Finkelstein, R. R., and Abrams, S. R. (2010). Abscisic acid: emergence of a core signaling network. Annu. Rev. Plant Biol. 61, 651-679. doi: 10.1146/annurev-arplant-042809-112122

Dong, H., Deng, Y., Mu, J., Lu, Q., Wang, Y., Xu, Y., et al. (2007). The Arabidopsis Spontaneous Cell Deathl gene, encoding a $\zeta$-carotene desaturase essential for carotenoid biosynthesis, is involved in chloroplast development, photoprotection and retrograde signalling. Cell Res. 17, 458-470. doi: $10.1038 /$ cr.2007.51

Havaux, M. (2014). Carotenoid oxidation products as stress signals in plants. Plant J. 79, 597-606. doi: 10.1111/tpj.12386

Lee, D.-K., and Sieburth, L. E. (2012). The bps signal: embryonic arrest from an auxin-independent mechanism in bypass triple mutants. Plant Signal. Behav. 7, 698-700. doi: $10.4161 / \mathrm{psb} .20282$

Lee, D.-K., Van Norman, J. M., Murphy, C., Adhikari, E., Reed, J. W., and Sieburth, L. E. (2012). In the absence of BYPASS1-related gene function, the bps signal disrupts embryogenesis by an auxin-independent mechanism. Development 139, 805-815. doi: 10.1242/dev.077313

Mittler, R., and Blumwald, E. (2015). The roles of ROS and ABA in systemic acquired acclimation. Plant Cell 27, 64-70. doi: 10.1105/tpc.114.133090

Park, H., Kreunen, S., Cuttriss, A. J., DellaPenna, D., and Pogson, B. J. (2002). Identification of the carotenoid isomerase provides insight into carotenoid biosynthesis, prolamellar body formation, and photomorphogenesis. Plant Cell 14, 321-332. doi: $10.1105 /$ tpc.010302

Ramel, F., Birtic, S., Cuiné, S., Triantaphylidès, C., Ravanat, J.-L., and Havaux, M. (2012a). Chemical quenching of singlet oxygen by carotenoids in plants. Plant Physiol. 158, 1267-1278. doi: 10.1104/pp.111.182394

Ramel, F., Birtic, S., Ginies, C., Soubigou-Taconnat, L., Triantaphylidès, C., and Havaux, M. (2012b). Carotenoid oxidation products are stress signals that mediate gene responses to singlet oxygen in plants. Proc. Natl. Acad. Sci. U.S.A. 109, 5535-5540. doi: 10.1073/pnas.1115982109

Ramel, F., Ksas, B., Akkari, E., Mialoundama, A. S., Monnet, F., Krieger-Liszkay, A., et al. (2013a). Light-induced acclimation of the Arabidopsis chlorinal mutant to singlet oxygen. Plant Cell 25, 1445-1462. doi: 10.1105/tpc.113. 109827

Ramel, F., Ksas, B., and Havaux, M. (2013b). Jasmonate: a decision maker between cell death and acclimation in the response of plants to singlet oxygen. Plant Signal. Behav. 8:e26655. doi: 10.4161/psb.26655

Ramel, F., Mialoundama, A. S., and Havaux, M. (2013c). Nonenzymic carotenoid oxidation and photooxidative stress signalling in plants. J. Exp. Bot. 64, 799-805. doi: 10.1093/jxb/ers223

Seto, Y., and Yamaguchi, S. (2014). Strigolactone biosynthesis and perception. Curr. Opin. Plant Biol. 21, 1-6. doi: 10.1016/j.pbi.2014.06.001

Shumbe, L., Bott, R., and Havaux, M. (2014). Dihydroactinidiolide, a high light-induced $\beta$-carotene derivative that can regulate gene expression and photoacclimation in Arabidopsis. Mol. Plant 7, 1248-1251. doi: $10.1093 / \mathrm{mp} / \mathrm{ssu} 028$

Triantaphylidès, C., and Havaux, M. (2009). Singlet oxygen in plants: production, detoxification and signaling. Trends Plant Sci. 14, 219-228. doi: 10.1016/j.tplants.2009.01.008

Van Norman, J. M., Frederick, R. L., and Sieburth, L. E. (2004). BYPASS1 negatively regulates a root-derived signal that controls plant architecture. Curr. Biol. 14 1739-1746. doi: 10.1016/j.cub.2004.09.045

Van Norman, J. M., and Sieburth, L. E. (2007). Dissecting the biosynthetic pathway for the bypass1 root-derived signal. Plant J. 49, 619-628. doi: 10.1111/j.1365313X.2006.02982.x

Van Norman, J. M., Zhang, J., Cazzonelli, C. I., Pogson, B. J., Harrison, P. J., Bugg, T. D. H., et al. (2014). Periodic root branching in Arabidopsis requires synthesis of an uncharacterized carotenoid derivative. Proc. Natl. Acad. Sci. U.S.A. 111, E1300-E1309. doi: 10.1073/pnas.1403016111

Waldie, T., McCulloch, H., and Leyser, O. (2014). Strigolactones and the control of plant development: lessons from shoot branching. Plant J. 79, 607-622. doi: $10.1111 /$ tpj. 12488

Conflict of Interest Statement: The author declares that the research was conducted in the absence of any commercial or financial relationships that could be construed as a potential conflict of interest.

Copyright $(2015$ Tian. This is an open-access article distributed under the terms of the Creative Commons Attribution License (CC BY). The use, distribution or reproduction in other forums is permitted, provided the original author(s) or licensor are credited and that the original publication in this journal is cited, in accordance with accepted academic practice. No use, distribution or reproduction is permitted which does not comply with these terms. 